\section{P044 REGIONAL DIFFERENCES IN USE OF GETCHECKEDONLINE AND CLIENT CHARACTERISTICS ACROSS BRITISH COLUMBIA, CANADA}

${ }^{1}$ Aidan Ablona*, ${ }^{2}$ Ellen Korol, ${ }^{2}$ Barbara Gauthier, ${ }^{3}$ Laurence Campeau, ${ }^{3}$ Sophie BannarMartin, ${ }^{2}$ Maja Karlsson, ${ }^{2}$ Lorena Hiscoe, ${ }^{3}$ Dee Hoyano, ${ }^{1}$ Devon Haag, ${ }^{1}$ Troy Grennan, ${ }^{1}$ Mark Gilbert. 'BC Centre for Disease Control, Clinical Prevention Services, Vancouver, Canada; ${ }^{2}$ Interior Health Authority, Kelowna, Canada; ${ }^{3}$ sland Health Authority, Victoria, Canada

10.1136/sextrans-2019-sti.249

Background GetCheckedOnline (GCO) launched in Vancouver, British Columbia (BC), Canada, in September 2014, offering online access to sexually-transmitted infection (STI) testing. In February 2016, the program expanded to smaller urban, suburban, and rural communities in south central BC (Interior Health Authority) and Vancouver Island (Island Health Authority). Given regional differences in STI clinic service availability, we used GCO program data to compare socio-demographic and behavioural measures by region among clients completing testing through GCO.

Methods GCO test episode and client-level data were included from the first 23 months of regional expansion (February 2016 to December 2018). Variables were analyzed descriptively. Bivariate analyses comparing Vancouver with each of the expansion regions (Island and Interior) were conducted using chi-squared tests; significant results $(\mathrm{p}<0.05)$ are listed below.

Results During the study period, 6,329 unique clients completed testing, with 3,435 (54\%) from Vancouver, 1,834 (29\%) from Island, and 1,060 (17\%) from Interior. In total, 10,953 test episodes were completed. STI positivity was higher in Interior compared to Vancouver $(6.1 \%$ vs $4.8 \%)$. Vancouver testers were older and a higher proportion identified as men who have sex with men (35\%) compared to Interior (14\%) and Island (26\%). Greater proportions of testers from the expansion regions were symptomatic and reported STI risk factors (contact with STI-positive partner, condomless sex with $>1$ partner) at time of testing. Higher proportions of testing events in expansion regions were reported as firsttime STI tests (never tested before: 23\% Interior and 15\% Island vs 9\% Vancouver).

Conclusion This study highlights important regional differences in socio-demographic and sexual risk behaviours among GCO clients. Further research describing predictors of STI positivity, repeat testing patterns, and differences in barriers to testing across regions will help contextualize the impact of an online STI testing service across urban, suburban, and rural environments.

Disclosure No significant relationships.

\section{P045 \\ IS CHLAMYDIA TESTING IN GENERAL PRACTICE SUSTAINED WHEN FINANCIAL INCENTIVES OR AUDIT + FEEDBACK ARE REMOVED: A CLUSTER RCT}

${ }^{1}$ Jane Hocking*, ${ }^{2}$ Anna Wood, ${ }^{1}$ Sabine Braat, ${ }^{1}$ Callum Jones, ${ }^{3}$ Meredith Temple-Smith, ${ }^{4}$ Mieke Van Driel, ${ }^{5}$ Matthew Law, ${ }^{6}$ Basil Donovan, ${ }^{7}$ Christopher Fairley, ${ }^{8}$ John Kaldor, ${ }^{9}$ Rebecca Guy, ${ }^{10}$ Nicola Low, ${ }^{11}$ Liliana Bulfone, ${ }^{3}$ Jane Gunn. 'University of Melbourne, Melbourne School of Population and Global Health, Carlton, Australia; ${ }^{2}$ University of Melbourne, Department of General Practice, Carlton, Australia; ${ }^{3}$ The University of Melbourne, General Practice, Melbourne, Australia; ${ }^{4}$ University of Queensland, Brisbane, Australia; ${ }^{5}$ University of New South Wales, Kirby Institute, Sydney, Australia; ${ }^{6}$ UNSW Sydney, Kirby Institute, Sydney, Australia; '7Melbourne Sexual Health Centre, Melbourne, Australia; ${ }^{8}$ University of New South Wales, The Kirby Institute for Infection and Immunity in Society, Kensington, Australia; ${ }^{9}$ Kirby institute, Sydney, Australia; ${ }^{10}$ University of Bern, Institute of Social and Preventive Medicine (ISPM), Bern, Switzerland; ${ }^{11}$ Deakin University, Melbourne, Australia

\subsection{6/sextrans-2019-sti.250}

Background Financial incentives (FI) and audit+feedback (AF) are often used to improve general practitioner (GP) performance. In the Australian Chlamydia Control Effectiveness Pilot (ACCEPt), a cluster-randomised controlled trial (RCT), GPs in the intervention arm received a FI of $\$ 5-\$ 8$ per chlamydia test and a quarterly AF report of chlamydia testing rates for their 16-29 year old patients. The objective of this present study was to examine the effects of removal of these measures on chlamydia testing rates.

Methods At the end of the ACCEPt trial, we designed a new 2X2 factorial cluster-RCT. ACCEPt intervention clinics were re-randomised to four arms: remove $\mathrm{AF} /$ retain $\mathrm{FI}$, remove $\mathrm{FI} /$ retain $\mathrm{AF}$, remove both $\mathrm{AF}$ and $\mathrm{FI}$, or retain both $\mathrm{FI}$ and $\mathrm{AF}$. The main comparisons were: removal vs. retention of FI and removal vs. retention of AF. The primary outcome was the absolute difference in chlamydia testing rates (proportion of 16-29 year old patients tested for chlamydia within a 12month period) at year 2 compared with baseline, estimated using mixed-effect logistic regression models accounting for clustering at the clinical level.

Results 55 clinics were re-randomised. Chlamydia testing decreased from $20.0 \%$ to $11.7 \%$ in clinics with FI removed and from $20.1 \%$ to $14.4 \%$ in clinics that retained FI, with no evidence of a treatment effect between arms (difference $=2.6 \%$; 95\%CI: $-0.1,5.7$ ). Testing decreased from $20.8 \%$ to $11.5 \%$ in clinics with $\mathrm{AF}$ reports removed and from $19.7 \%$ to $14.8 \%$ in clinics that retained $\mathrm{AF}$, with a larger reduction for removal than for retention of $\mathrm{AF}$ (difference $=4.4 \%$ (1.1, 7.8).

Conclusion Chlamydia testing rates declined in all clinics after the end of ACCEPt. Chlamydia testing rates fell more when quarterly audit+feedback reports were removed than when financial incentives were removed. Policy makers and clinicians should be aware of the challenge to sustaining chlamydia testing uptake in GP clinics.

Disclosure No significant relationships. 